\title{
Redox-Modulated Recognition of Tetrazines using Thioureas
}

\author{
Brian J. Jordan ${ }^{\dagger}$, Michael A. Pollier ${ }^{\dagger}$, Luke A. Miller ${ }^{\dagger}$, Christopher \\ Tiernan $^{\dagger}$, Gilles Clavier ${ }^{\ddagger}$, Pierre Audebert ${ }^{\ddagger}$, and Vincent M. Rotello ${ }^{\dagger \star}$ \\ 'Department of Chemistry, University of Massachusetts, Amherst, Massachusetts, \\ 01003, and PPSM, ENS Cachan, CNRS, UniverSud, 61 av President Wilson, F-94230 \\ CACHAN, France
}

rotello@chem.umass.edu

\section{Supporting Information}

\section{Materials and General Methods}

All chemicals and solvents were purchased from Aldrich Chemical Co., and used as received unless otherwise specified. Tetrabutylammonium perchlorate (TBAP, obtained from SACHEM. Electrometric grade) was dissolved in $\mathrm{CHCl}_{3}$, washed with distilled water, recrystallized twice from ethyl acetate and dried for several days under vacuum. Tetrazines $\mathbf{1}$ and $\mathbf{2}$ were synthesized according to previously reported procedures ${ }^{1}$ utilizing the precursor designed by Hiskey et $a{ }^{2}{ }^{2}$ 


\section{${ }^{1} \mathrm{H}$ NMR titrations}

All titrations were performed on a Bruker $400 \mathrm{MHz} \mathrm{NMR}$ spectrometer in $\mathrm{CDCl}_{3}$, using $1 \mathrm{mM}$ tetrazine as a constant host solution and a $50 \mathrm{mM}$ stock solution of thiourea 3 guest.

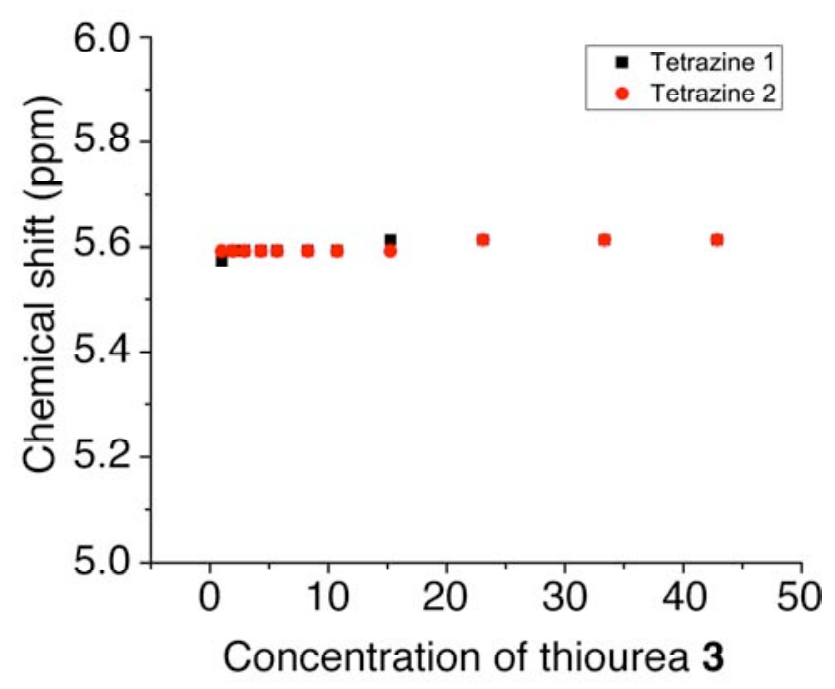

Only slight chemical shifts (above) were recorded for the titration of tetrazines with thiourea $\mathbf{3}$ suggesting that there was minimal association between tetrazines and thiourea in the neutral state. Protons monitored at $\sim 5.6 \mathrm{ppm}(\mathrm{br}, 2 \mathrm{H})$ correspond to hydrogen bond donors in thiourea. However, the small proton peak broadening (usually indicative of hydrogen bonding) observed in the ${ }^{1} \mathrm{H}$ NMR spectra required further experimentation to determine the existence of hydrogen bonding within the supramolecular complex.

\section{Fluorescence titrations}

Aliquots of thiourea guest $(100 \mathrm{mM})$ were added to a solution of tetrazine $\mathbf{1}$ maintaining a constant host concentration $(1 \mathrm{mM})$ in $\mathrm{CHCl}_{3}$ similar to previous NMR titrations. Spectra were recorded after each addition on a Shimadzu RF 5301 PC 
spectrofluorophotometer using $\lambda_{\mathrm{ex}}=540 \mathrm{~nm}$ and scanning over a range of $545-650 \mathrm{~nm}$. Intensity at $\lambda_{\max }=570 \mathrm{~nm}$ versus concentration yielded an inverse Stern-Volmer plot.

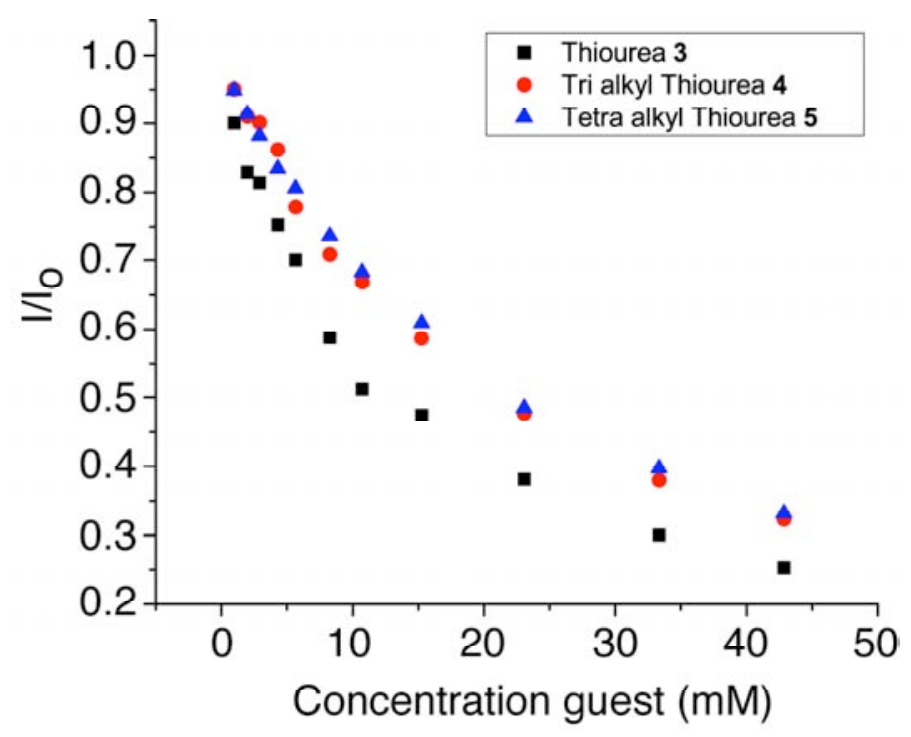

Inverse-Stern Volmer plot (above) demonstrated quenching of tetrazine $\mathbf{1}$ by various thiourea derivatives (3-5). Control trialkyl thiourea 4 and tetra alkyl thiourea 5 do not participate in two point hydrogen bonding, but still exhibit quenching of $\mathbf{1}$. The quenching mechanism is a result of electron donation from the sulfur atom in the thiourea to the electron deficient tetrazine core and is consistent with previous results. ${ }^{1}$ Subtraction of the electron donation from thiourea $\mathbf{3}$ yields only a minimal contribution from hydrogen bonding. Therefore, there is relatively no complex formation in the oxidized state between tetrazines and thiourea 3 .

\section{Electrochemistry}

All electrochemical experiments were performed using a Cypress System potentiostat in $\mathrm{CH}_{2} \mathrm{Cl}_{2}$ with $0.1 \mathrm{M} \mathrm{TBAP}$ as the supporting electrolyte. The argon-purged temperature controlled cell consisted of a three electrode system with a $1 \mathrm{~mm}$ platinum button as the 
working electrode, a gold-platted electrode as the auxiliary electrode, and a silver wire pseudo reference electrode. Bis(pentamethylcyclopentadienyl)iron (II) was added as an internal standard and all potentials were referenced versus the ferrocenium/ ferrocene redox couple. Excess thiourea (50 equivalents) was added to $1 \mathrm{mM}$ solutions of tetrazine and degassed by bubbling argon through the solution for 10 minutes. Both cyclic voltammetry and square waves were employed to quantify the corresponding shift in the $\mathrm{E}_{1 / 2}$ of the unbound and bound species.
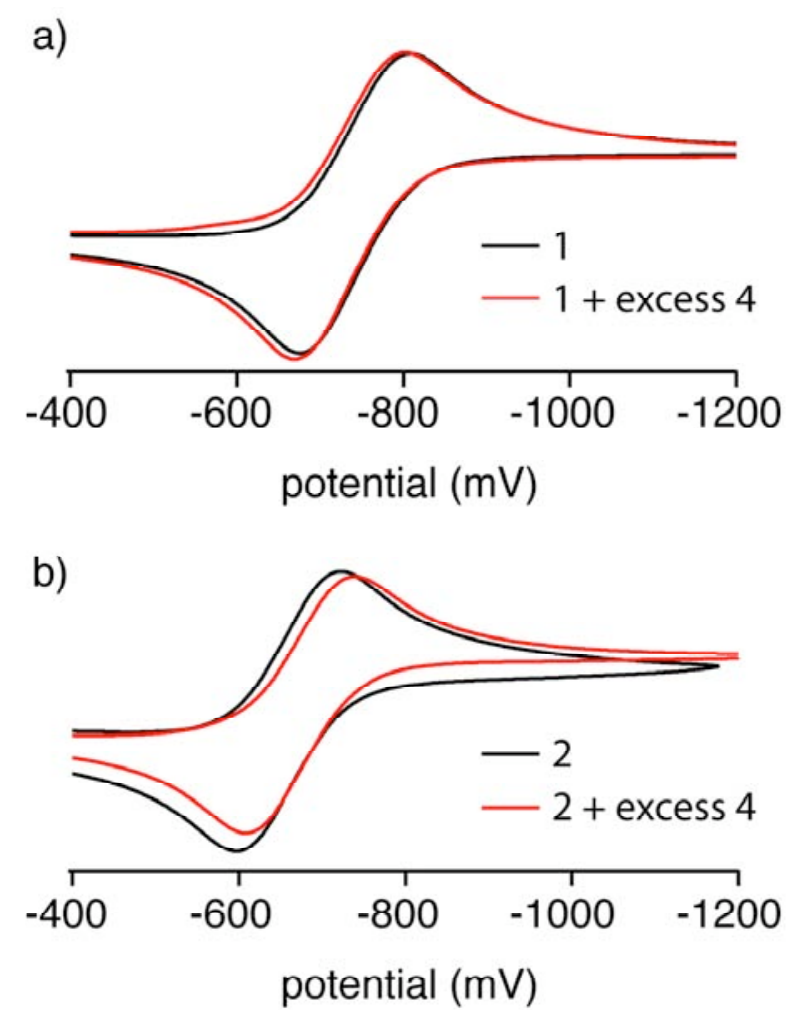

As a control, an excess of trialkyl thiourea $\mathbf{4}$ was added to a solution of tetrazine $\mathbf{1}$ (a) and 2 (b). No shift in the $\mathrm{E}_{1 / 2}$ was recorded for both tetrazine derivatives as expected since trialkyl thiourea $\mathbf{4}$ does not participate in two point hydrogen bonding. 


\section{Computational Methods}

Tetrazines, diethyl thiourea, and their respective complexes were first optimized with HF $6-31 G^{*}$ to yield the minimum energy conformation. Single point energies were calculated via DFT B3LYP 6-31G* and used to map the electrostatic potentials and $\Delta \mathrm{E}_{\text {calc }}$. Frequency calculations demonstrated positive values for all optimized structures. All calculations were performed using Jaguar v8.0 beta from Schrodinger, Inc.

(1) Audebert, P.; Miomandre, F.; Clavier, G.; Vernieres, M. C.; Badre, S.; MealletRenault, R. Chem. Eur. J. 2005, 11, 5667

(2) Coburn, M. D.; Buntain, G. A.; Harris, B. W.; Hiskey, M. A.; Lee, K. Y.; Ott, D. G. J. Heterocyclic Chem. 1991, 28, 2049. 